\title{
Ohmic Reservoir-based non-Markovianity and Quantum Speed Limit Time
}

\author{
Hong-Mei Zou ${ }^{1}$ * Rongfang Liu ${ }^{1}$, Dan Long ${ }^{1}$, Jianhe Yang ${ }^{1}$, and Danping Lin ${ }^{2}$ \\ ${ }^{1}$ Synergetic Innovation Center for Quantum Effects and Application, \\ Key Laboratory of Low-dimensional Quantum Structures and Quantum Control of Ministry of Education, \\ School of Physics and Electronics, Hunan Normal University, Changsha, 410081, People's Republic of China. \\ ${ }^{2}$ Faculty of Science, Guilin University of Aerospace Technology, Guilin 541004, People's Republic of China.
}

\begin{abstract}
We study the non-Markovianity and quantum speedup of a two-level atom (quantum system of interest) in a dissipative Jaynes-Cumming model, where the atom is embedded in a single-mode cavity, which is leaky being coupled to an external reservoir with Ohmic spectral density. We obtain the non-Markovianity characterized by using the probability of the atomic excited state and the negative decoherence rate in the time-local master equation. We also calculate the quantum speed limit time (QSLT) of the evolution process of the atom. The results show that, the atomcavity coupling is the main physical reasons of the transition from Markovian to non-Markovian dynamics and the transition from no speedup to speedup process, and the critical value of this sudden transition only depends on the Ohmicity parameter. The atom-cavity coupling and the appropriate reservoir parameters can effectively improve the non-Markovianity in the dynamics process and speed up the evolution of the atom. Moreover, the initial non-Markovian dynamics first turns into Markovian and then back to non-Markovian with increasing the atom-cavity coupling under certain condition. Finally, the physical interpretation is provided.
\end{abstract}

PACS numbers: 03.65.Yz, 03.67.Lx, 42.50.-p, 42.50.Pq.

Keywords: Quantum Speed Limit Time, non-Markovianity, dissipative cavity

\section{INTRODUCTION}

As we known, the decoherence effect and the energy dissipation caused by coupling of system-environment will bring remarkable influences on the dynamical behaviour of the open system. The evolution process of the open system is Markovian if a quantum system is weakly coupled to a memoryless environment, while the evolution process is non-Markovian if a quantum system is strong coupled to a memory environment [1 6 . The non-Markovian effect in the dynamics process can be described by the non-Markovianity. Many efforts have been made to define non-Markovianity, to measure it, and to take advantage of it [7 25]. For examples, the nonmarkovianity was quantified by correlations in Ref. [10, the authors in [21, 24] studied the measurement of nonMarkovianity and Paternostro's team studied geometrical characterization of non-Markovianity 17. In recent years, the research on non-Markovianity in the dynamics process of the open system has attracted the attention of the community, both theoretically and experimentally 26] 29].

On the other hand, quantum speed limit (QSL) has been considered a purely quantum phenomenon with no corresponding concept in classical mechanics, which sets a bound on the maximal evolution velocity that a quantum system needs to evolve between two distinguishable states. Driving a given initial state to a target state at the maximal evolution speed is one of fundamental and important tasks of quantum physics, thus QSL plays a significant role in the fields of quantum computation,

\footnotetext{
*zhmzc1997@hunnu.edu.cn
}

quantum metrology, and so on 30 34. The minimal evolution time between two distinguishable states of a quantum system is defined as the quantum speed limit time(QSLT) 35 39. For closed systems, the QSLT is defined by $\tau=\max \left\{\frac{\pi \hbar}{2 \Delta E}, \frac{\pi \hbar}{2 E}\right\}$, where $\Delta E$ in MandelstamTamm(MT) bound [40] and $E$ in Margolus-Levitin(ML) bound [4] are the fluctuation and the mean value of the initial-state energy, respectively. For open systems, Deffner and Lutz obtained the unified bound of QSLT from the MT and ML types by using the Bures angle and showed that the non-Markovian effects could speed up the quantum evolution 42. In recent years, many efforts have been made in the study of QSLT of an open system [43-50].

In addition, much valuable effort have also been devoted to the relationships between the non-Markovianity and the QSL [50 56], such as quantum speedup in a memory environment [51, quantum speedup in open quantum systems [52, 53] and the relationship between the quantum speedup and the formation of a systemenvironment bound state [50, 54. The authors in 57. found that a classical field can effectively regulate the non-Markovianity and the QSLT of an open qubit. Namely, the strong coupling of qubit-environment and an external classical field can all realize the transformation from Markovian to non-Markovian dynamics and the speedup evolution of the system. In these studies mentioned above, the environment is usually at zero temperature and has generally the Lorentzian spectral density.

In Ref. 58, we have studied the QSLT and the nonMarkovianity of the atom in Jaynes-Cummings model coupling with the Lorentzian reservoir and the Ohmic reservoir with a LorentzDrude cutoff function, respectively, and the reservoir is at zero temperature, and we characterized the non-Markovianity by using the posi- 
tive derivative of the trace distance. However, in this paper, we focus on the Ohmic reservoir and the master equation of the atom-cavity subsystem interacting with the reservoir at $T$ temperature, and we characterize the non-Markovianity by using the probability of the atomic excited state and the negative decoherence rate in the time-local master equation. The results show that the atom-cavity coupling and the appropriate reservoir parameters can improve the non-Markovianity in the dynamics process and accelerate the evolution of the atom.

The outline of the paper is the following. In Section II, we describe a physical model. In Section III, we introduce the non-Markovianity and the quantum speed limit time. Results and discussions are provided in Section IV. Finally, we give a brief summary in Section V.

\section{PHYSICAL MODEL}

We consider a dissipative Jaynes-Cummings model [59, 60., namely, an atom is in a leaky cavity that the leakage is usually modelled by coupling of the cavity mode to the bosonic modes of the reservoir. The Hamiltonian of the total system is given by $(\hbar=1)$

$$
\hat{H}=\hat{H}_{J C}+\hat{H}_{C R}
$$

here

$$
\hat{H}_{J C}=\frac{1}{2} \omega_{0} \hat{\sigma}_{z}+\omega_{0} \hat{a}^{\dagger} \hat{a}+\Omega\left(\hat{a} \hat{\sigma}_{+}+\hat{a}^{\dagger} \hat{\sigma}_{-}\right)
$$

and

$$
\hat{H}_{C R}=\sum_{k} \omega_{k} \hat{b}_{k}^{\dagger} \hat{b}_{k}+\left(\hat{a}+\hat{a}^{\dagger}\right) \sum_{k} g_{k}\left(\hat{b}_{k}^{\dagger}+\hat{b}_{k}\right)
$$

where the atomic transition frequency is $\omega_{0}$ and the Pauli matrices of the atom are $\hat{\sigma}_{z}$ and $\hat{\sigma}_{ \pm} \cdot \hat{a}^{\dagger}(\hat{a})$ and $\hat{b}_{k}^{\dagger}\left(\hat{b}_{k}\right)$ express the creation(annihilation) operators of the cavity and the $k$-th mode of reservoir with the frequency $\omega_{k}$, respectively. $\Omega$ and $g_{k}$ are the coupling strength of the atom-cavity and the cavity-reservoir, respectively.

In this work, we suppose that the total number of excitations is $n=1$ in the total system. The eigenstates and eigenvalues of the Hamiltonian $\hat{H}_{J C}$ are given by $\left|\varphi_{1, \pm}\right\rangle=\frac{1}{\sqrt{2}}(|1, g\rangle \pm|0, e\rangle)$ and $E_{1, \pm}=\frac{1}{2} \omega_{0} \pm \Omega$ for $n=1$, while the ground state and the corresponding energy eigenvalue are $\left|\varphi_{0}\right\rangle=|0, g\rangle$ and $E_{0}=-\frac{1}{2} \omega_{0}$. Then we assume that $\hat{A}_{1}^{+}=\left|\varphi_{1,-}\right\rangle\left\langle\varphi_{0}\right|$ and $\hat{A}_{1}^{-}=\left|\varphi_{0}\right\rangle\left\langle\varphi_{1,-}\right|$ are the jump operators between $\left|\varphi_{1,-}\right\rangle$ and $\left|\varphi_{0}\right\rangle$, and $\hat{A}_{2}^{+}=\left|\varphi_{1,+}\right\rangle\left\langle\varphi_{0}\right|$ and $\hat{A}_{2}^{-}=\left|\varphi_{0}\right\rangle\left\langle\varphi_{1,+}\right|$ are the jump operators between $\left|\varphi_{1,+}\right\rangle$ and $\left|\varphi_{0}\right\rangle$. Performing the BornMarkov and the rotating wave approximations, tracing out the freedom degrees of the reservoir in the interaction picture and then going back to the Schrödinger picture, we can obtain the master equation for the atom-cavity subsystem interacting with the reservoir at $T$ temperature as follows [61, 62]:

$$
\begin{aligned}
\frac{d}{d t} \varrho(t) & =-i\left[\hat{H}_{J C}, \varrho(t)\right] \\
& +\frac{1}{2} \gamma\left(\omega_{1}, t\right)\left(\hat{A}_{1}^{-} \varrho(t) \hat{A}_{1}^{+}-\frac{1}{2}\left\{\hat{A}_{1}^{+} \hat{A}_{1}^{-}, \varrho(t)\right\}\right) \\
& +\frac{1}{2} \gamma\left(\omega_{2}, t\right)\left(\hat{A}_{2}^{-} \varrho(t) \hat{A}_{2}^{+}-\frac{1}{2}\left\{\hat{A}_{2}^{+} \hat{A}_{2}^{-}, \varrho(t)\right\}\right) \\
& +\frac{1}{2} \gamma\left(-\omega_{1}, t\right)\left(\hat{A}_{1}^{+} \varrho(t) \hat{A}_{1}^{-}-\frac{1}{2}\left\{\hat{A}_{1}^{-} \hat{A}_{1}^{+}, \varrho(t)\right\}\right) \\
& +\frac{1}{2} \gamma\left(-\omega_{2}, t\right)\left(\hat{A}_{2}^{+} \varrho(t) \hat{A}_{2}^{-}-\frac{1}{2}\left\{\hat{A}_{2}^{-} \hat{A}_{2}^{+}, \varrho(t)\right\}\right)
\end{aligned}
$$

here $\omega_{1,2}=\omega_{0} \mp \Omega$ is the transition frequency of the dressed-states $\left|\varphi_{1, \mp}\right\rangle \leftrightarrow\left|\varphi_{0}\right\rangle . \quad \gamma\left(\omega_{1}, t\right)$ and $\gamma\left(\omega_{2}, t\right)$ are the time dependent decay rates for $\left|\varphi_{1,-}\right\rangle$ and $\left|\varphi_{1,+}\right\rangle$, respectively, i.e.

$$
\gamma\left(\omega_{j}, t\right)=2 \Re\left[\int_{0}^{t} d \tau \int_{-\infty}^{+\infty} d \omega e^{i\left(\omega_{j}-\omega\right) \tau} J(\omega)\right]
$$

in which $J(\omega)$ is the spectral density of the reservoir and

$$
\gamma\left(-\omega_{j}, t\right)=\exp \left(-\frac{\omega_{j}}{k_{B} T}\right) \gamma\left(\omega_{j}, t\right)
$$

For simplicity, we only discuss the reservoir at zero temperature 63] in the following. Eq. (4) becomes

$$
\begin{aligned}
\frac{d}{d t} \varrho(t) & =-i\left[\hat{H}_{J C}, \varrho(t)\right] \\
& +\frac{1}{2} \gamma\left(\omega_{1}, t\right)\left(\hat{A}_{1}^{-} \varrho(t) \hat{A}_{1}^{+}-\frac{1}{2}\left\{\hat{A}_{1}^{+} \hat{A}_{1}^{-}, \varrho(t)\right\}\right) \\
& +\frac{1}{2} \gamma\left(\omega_{2}, t\right)\left(\hat{A}_{2}^{-} \varrho(t) \hat{A}_{2}^{+}-\frac{1}{2}\left\{\hat{A}_{2}^{+} \hat{A}_{2}^{-}, \varrho(t)\right\}\right)
\end{aligned}
$$

We can acquire an analytical solution of the density operator $\varrho(t)$ in the dressed-state basis $\left\{\left|\varphi_{0}\right\rangle,\left|\varphi_{1,-}\right\rangle,\left|\varphi_{1,+}\right\rangle\right\}$ from Eq. (7), then the density matrix $\rho(t)$ of the atom in the standard basis $\{|e\rangle,|g\rangle\}$ is also obtained by means of the representation transformation and taking a partial trace over the freedom degrees of the cavity. Suppose the initial state is $\left\{\rho_{11}(0), \rho_{10}(0), \rho_{01}(0), \rho_{00}(0)\right\}$, the density matrix $\rho(t)$ [58] of the atom at all time $t$ is expressed as

$$
\rho(t)=\left(\begin{array}{cc}
|p(t)|^{2} \rho_{11}(0) & p(t) \rho_{10}(0) \\
p(t)^{*} \rho_{01}(0) & 1-|p(t)|^{2} \rho_{11}(0)
\end{array}\right)
$$

where the probability amplitude $p(t)$ can be given by

$$
p(t)=\frac{1}{2} \sum_{j=1}^{2} e^{-i \omega_{j} t} e^{-\frac{1}{4} \beta_{j}}
$$

here

$$
\beta_{j}=\int_{0}^{t} \gamma\left(\omega_{j}, t^{\prime}\right) d t^{\prime}
$$

Considering the structured reservoir with an Ohmic spectral density

$$
J(\omega)=\eta \omega^{s} \omega_{c}^{1-s} e^{-\omega / \omega_{c}}
$$


where $s$ is the Ohmicity parameter, which moves the spectrum from sub-Ohmic $(0<s<1)$ to Ohmic if $(s=1)$ and super-Ohmic $(s>1)$ regimes 64 66. $\omega_{c}$ and $\eta$ being the cut-off frequency and the dimensionless coupling constant, which are related to the reservoir correlation time $\tau_{B}$ and the relaxation time $\tau_{R}$ (over which the state of the system changes in the Markovian limit of a flat spectrum) by $\tau_{B} \approx \omega_{c}^{-1}$ and $\tau_{R} \approx \eta^{-1} . \omega_{c}<\omega_{0}$ implies that the spectrum of the reservoir does not completely overlap with the frequency of the cavity, that is, the reservoir is effectively adiabatic, so that the evolution behaviour of the system is essentially non-Markovian. While $\omega_{c}>\omega_{0}$ indicates the converse case, which the quantum information is quickly dissipated, the evolution behaviour of the system is Markovian. The smaller the value of $\eta$ is, the longer the reservoir correlation time is, and the more obvious the non-Markovian effect is 67 69.

Common values of $s$ are $\frac{1}{2}, 1$ and 3, inserting Eq. 111 into Eq. (5), $\gamma\left(\omega_{j}, t\right)$ is written as

$$
\begin{aligned}
s=\frac{1}{2}: \gamma\left(\omega_{j}, t\right) & =-\frac{2 \eta \omega_{c} \sqrt{\pi}}{\left(1+\omega_{c}^{2} t^{2}\right)^{\frac{1}{4}}} \sin \left(\omega_{j} t-\frac{\alpha_{0}}{2}\right) \\
s=1: \gamma\left(\omega_{j}, t\right) & =-\frac{2 \eta \omega_{c}}{\left(1+\omega_{c}^{2} t^{2}\right)^{\frac{1}{2}}} \sin \left(\omega_{j} t-\alpha_{0}\right) \\
s=3: \gamma\left(\omega_{j}, t\right) & =-\frac{2 \eta \omega_{j}^{2}}{\omega_{c}\left(1+\omega_{c}^{2} t^{2}\right)^{\frac{1}{2}}} \sin \left(\omega_{j} t-\alpha_{0}\right) \\
& -\frac{2 \eta \omega_{j}}{\left(1+\omega_{c}^{2} t^{2}\right)} \sin \left(\omega_{j} t-2 \alpha_{0}\right) \\
& -\frac{4 \eta \omega_{c}}{\omega_{c}\left(1+\omega_{c}^{2} t^{2}\right)^{\frac{3}{2}}} \sin \left(\omega_{j} t-3 \alpha_{0}\right)
\end{aligned}
$$

with $\alpha_{0}=\arctan \left(\omega_{c} t\right)$.

We can not get the analytical expressions for $\beta_{j}$ from Eq. (10) and Eqs. (12)-(14), but we can calculate mathematically $\beta_{j}$ for the sub-Ohmic, Ohmic and super-Ohmic spectra, respectively.

In view of Eq. (8), we can also write a time-local master equation [70] for the density operator $\rho(t)$ as

$$
\begin{aligned}
\frac{d}{d t} \rho(t) & =\mathcal{L} \rho(t) \\
& =-\frac{i}{2} S(t)\left[\hat{\sigma}_{+} \hat{\sigma}_{-}, \rho(t)\right]+\Gamma(t)\left\{\hat{\sigma}_{-} \rho(t) \hat{\sigma}_{+}\right. \\
& \left.-\frac{1}{2} \hat{\sigma}_{+} \hat{\sigma}_{-} \rho(t)-\frac{1}{2} \rho(t) \hat{\sigma}_{+} \hat{\sigma}_{-}\right\}
\end{aligned}
$$

where the Lamb frequency shift $S(t)$ and the decoherence rate $\Gamma(t)$ can be respectively expressed as

$$
S(t)=-2 \Im\left[\frac{\dot{p}(t)}{p(t)}\right]
$$

and

$$
\Gamma(t)=-2 \Re\left[\frac{\dot{p}(t)}{p(t)}\right]
$$

$S(t)$ describes the contribution from the unitary part of the evolution under dynamical decoherence. $\Gamma(t)$ characterizes the dissipation and the feedback of the information of the system. $\Gamma(t)>0$ indicates that quantum information flows from the system to its environment, i.e. Markovian process. $\Gamma(t)<0$ expresses that quantum information flows back from its environment to the system, i.e. non-Markovian process.

\section{NON-MARKOVIANITY AND QUANTUM SPEED LIMIT TIME}

\section{A. Non-Markovianity}

In the dynamics process of an open system, the nonMarkovianity can describe the total backflow of information to the system from its environment. Among the different measurement of the non-Markovianity, the method based on the time rate of change of the trace distance is more commonly used at present. The trace distance between $\rho_{1}(t)$ and $\rho_{2}(t)$ is defined as $\mathcal{D}\left(\rho_{1}(t), \rho_{2}(t)\right)=\frac{1}{2} \operatorname{Tr}\left\|\rho_{1}(t)-\rho_{2}(t)\right\|$, which expresses the distinguishability between the two states $\rho_{1,2}(t)$ evolving from their respective initial forms $\rho_{1,2}(0)$ [8]. The time rate of change of the trace distance can be expressed as $\sigma\left(t, \rho_{1,2}(0)\right)=\frac{d}{d t} \mathcal{D}\left(\rho_{1}(t), \rho_{2}(t)\right) . \quad \sigma\left(t, \rho_{1,2}(0)\right)<0$ indicates that $\mathcal{D}\left(\rho_{1}(t), \rho_{2}(t)\right)$ decreases with time because the information flows irreversibly from the system to the environment, $\sigma\left(t, \rho_{1,2}(0)\right)>0$ shows that $\mathcal{D}\left(\rho_{1}(t), \rho_{2}(t)\right)$ is no longer decreasing monotonously because the information backflow from the environment to the system. The non-Markovianity can be calculated by $\mathcal{N}=\max _{\rho_{1,2}} \int_{\sigma>0} \sigma\left(t, \rho_{1,2}(0)\right) d t$ [52, 71]. If $\sigma\left(t, \rho_{1,2}(0)\right)<0, \mathcal{N}=0$ and the dynamics process of the system is Markovian. If $\sigma\left(t, \rho_{1,2}(0)\right)>0, \mathcal{N}>0$ and the dynamics process of the system is non-Markovian.

For the atom in Eq. (8), it has been proven that the optimal pair of initial states to maximize $\mathcal{N}$ are $\rho_{1}(0)=$ $|e\rangle\langle e|$ and $\rho_{2}(0)=|g\rangle\langle g|$ [37, 42]. Therefor the trace distance and its time rate of change are

$$
\mathcal{D}\left(\rho_{1}(t), \rho_{2}(t)\right)=|p(t)|^{2}
$$

and

$$
\sigma\left(t, \rho_{1,2}(0)\right)=\frac{d}{d t}|p(t)|^{2}
$$

From Eq. 17) and Eq. 19, the decoherence rate $\Gamma(t)$ can be obtained

$$
\Gamma(t)=-\frac{\sigma}{|p(t)|^{2}}
$$

Therefore, the non-Markovianity can be characterized by using the probability of the atomic excited state and the negative decoherence rate in the time-local master equation as

$$
\mathcal{N}=-\int_{\Gamma(t)<0}|p(t)|^{2} \Gamma(t) d t
$$


that is, there is non-Markovian in the dynamical process if the decoherence rate $\Gamma(t)$ is negative because the probability $|p(t)|^{2}$ is non negative. In the dissipative JC model, the quantum information will be exchanged between the reservoir with the cavity and between the cavity with the atom. Because we only care about the dynamics of the atom, both the cavity and its outside reservoir are regarded as the atomic environment. From Eqs. (9) and (20)-(21), we can see that the non-Markovianity $\mathcal{N}$ is determined by all environment parameters (including the atom-cavity coupling $\Omega$, the cavity-reservoir coupling $\eta$, the cut-off frequency $\omega c$ and the value of $s$ ). The non-Markovianity $\mathcal{N}$ is larger, the information from the environment feeding back to the atom is more.

\section{B. Quantum speed limit time}

The bound of the minimal evolution time from an initial state $\rho(0)$ to a final state $\rho(\tau)$ is defined as the quantum speed limit time (QSLT) of a syetem, where $\tau$ is an actual evolution time. If the initial state is $\rho(0)=\left|\psi_{0}\right\rangle\left\langle\psi_{0}\right|$ and its target state $\rho(\tau)$ satisfies the master equation $\dot{\rho}(t)=\mathcal{L} \rho(t)$ (see Eq. 115) with $\mathcal{L}$ being the positive generator of the dynamical semigroup, the QSLT can expressed as $\tau_{Q S L}=\sin ^{2} \beta[\rho(0), \rho(\tau)] / \Lambda_{\tau}^{\infty}$ according to the unified lower bound derived by Deffner and Lutz, where $\beta[\rho(0), \rho(\tau)]=\arccos \sqrt{\left\langle\psi_{0}\left|\rho_{\tau}\right| \psi_{0}\right\rangle}$ indicates the Bures angle between $\rho(0)$ and $\rho(\tau)$, and $\Lambda_{\tau}^{\infty}=\tau^{-1} \int_{0}^{\tau}\|\mathcal{L} \rho(t)\| d t$ with the operator norm $\|B\|$ equal to the largest eigenvalue of $\sqrt{B^{\dagger} B}$ 42. When $\rho(0)=|e\rangle\langle e|$, we can obtain the QSLT from Eq. (8) as

$$
\frac{\tau_{Q S L}}{\tau}=\frac{1-|p(t)|^{2}}{\int_{0}^{\tau} \partial_{t}|p(t)|^{2} d t}
$$

For the dynamics process from $\rho(0)$ to $\rho(\tau)$, the nonMarkovianity is also written as

$$
\mathcal{N}=\frac{1}{2}\left[\left.\left.\int_{0}^{\tau}\left|\partial_{t}\right| p(t)\right|^{2}|d t+| p(\tau)\right|^{2}-1\right]
$$

From Eqs. 222)- 23), the relationship [37] between the QSLT and the non-Markovianity can be obtained as

$$
\frac{\tau_{Q S L}}{\tau}=\frac{1-|p(\tau)|^{2}}{1-|p(\tau)|^{2}+2 \mathcal{N}}
$$

Eq. (24) shows that the QSLT is equal to the actual evolution time when $\mathcal{N}=0$, but the QSLT is smaller than the actual evolution time when $\mathcal{N}>0$. That is, the nonMarkovianity in the dynamics process can lead to the faster quantum evolution and the smaller QSLT.

\section{RESULTS AND DISCUSSIONS}

In this section, we analyse the relations between the trace distance with its derivative, between the non-

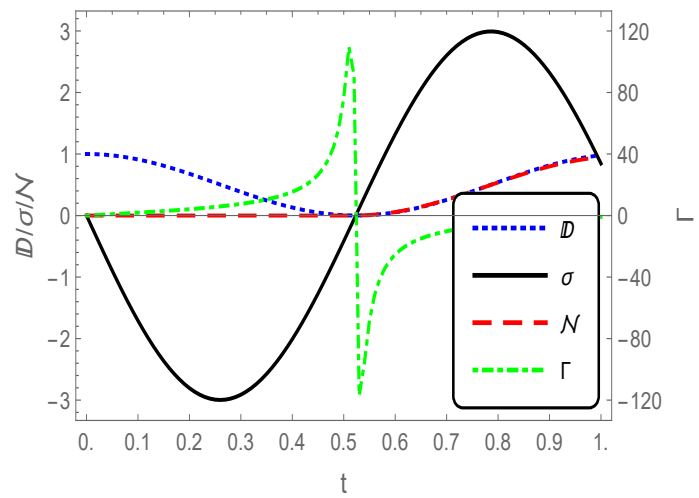

FIG. 1. (Color online) The dynamical curve of the trace distance and its derivative, the decoherence rate and the nonMarkovianity when $s=1$ (Ohmic spectrum), $\Omega=3 \omega_{0}$ and $\frac{\omega_{c}}{\omega_{0}}=2$. The left coordinate is $\mathcal{D}(t), \sigma(t)$ and $\mathcal{N}(t)$ while the right coordinate is $\Gamma(t)$. The blue dotted line is the trace distance $\mathcal{D}(t)$, the black solid line is the derivative $\sigma(t)$ of the trace distance, the red dashed line is the non-Markovianity $\mathcal{N}$, and the green dotted-dashed line is the decoherence rate $\Gamma(t)$. The other parameters are $\eta=0.1, \omega_{0}=1$ and $\omega_{c}=2$.

Markovianity with the decoherence rate and the derivative of the trace distance, as well as between the nonMarkovianity with the quantum speed limit time. We also study the influence of the atom-cavity coupling and the reservoir parameters on the the non-Markovianity and the quantum speed limit time.

In Fig.1, we draw the curve of the trace distance and its derivative, the decoherence rate and the nonMarkovianity when $s=1$ (Ohmic spectrum), $\Omega=3 \omega_{0}$ and $\frac{\omega_{c}}{\omega_{0}}=2$. We find that, the trace distance degenerates to zero from 1.0, and the derivative of the trace distance becomes negative and the decoherence rate simultaneously increases from zero. Then the trace distance again increases from zero, and the derivative of the trace distance becomes positive and the decoherence rate suddenly becomes negative at the same time. In addition, we can see that the non-Markovianity is equal to zero when the decoherence rate is positive (i.e. the derivative of the trace distance is negative), in which the quantum information flows from the system to its environment due to the dissipation of environment. The non-Markovianity is larger than zero when the decoherence rate is negative (i.e. the derivative of the trace distance is positive), where the quantum information flows back from its environment to the system because of the memory and feedback of environment. Therefore, once $\mathcal{D}(t)$ increases, the positive value of $\sigma(t)$ and the negative value of the decoherence rate will appear at the same time, the non-Markovianity in the dynamics process can be witnessed.

Fig.2(a) shows the dynamical properties of the derivative of the trace distance under different atom-cavity coupling $\Omega . \quad \sigma(t)$ changes from zero to negative when $\Omega=\omega_{0}$, thus the shaded area with positive $\sigma(t)$ is miss- 
ing which means $\mathcal{D}(t)$ is nonincreasing during the whole evolution, shown as the green dotted line in Fig.2(a). $\sigma(t)$ changes from zero to negative and then again to zero when $\Omega=1.55 \omega_{0}$, shown as the brown dashed line in Fig.2(a), the shaded area with positive $\sigma(t)$ is still zero but this is a threshold, that is, the shaded area with positive $\sigma(t)$ is appearing if $\Omega>1.55 \omega_{0}$. When $\Omega=3 \omega_{0}$, the red solid line changes from zero to negative and then to positive which means $\mathcal{D}(t)$ decreases first and then increases during the whole evolution, the shaded area with positive $\sigma(t)$ is $\int_{\sigma>0} \sigma\left(t, \rho_{1,2}(0)\right) d t=0.945$, as shaded in Fig.2(a). The non-Markovianity $\mathcal{N}$ is plotted in Fig.2(b) versus $\Omega / \omega_{0}$. For the region with $\Omega<1.55 \omega_{0}, \mathcal{N}$ is always zero, which means the derivative $\sigma(t)$ can never give a positive value, an example with $\Omega=\omega_{0}$ is the green $\operatorname{dot}(\mathcal{N}=0)$ in Fig.2(b) corresponding to the shaded area with positive $\sigma(t)$ of the green line in Fig.2(a). For the region with $\Omega>1.55 \omega_{0}$, there always exists a positive value of $\mathcal{N}$, the red $\operatorname{dot}(\mathcal{N}=0.948)$ in Fig.2(b) epresents an example of $\Omega=3 \omega_{0}$ which corresponds to the shaded area with the red line in Fig.2(a). The critical point with $\Omega=1.55 \omega_{0}$ shows the situation of the transition from Markovianity to non-Markovianity, which the brown dot $(\mathcal{N}=0)$ in Fig.2(b) corresponds to the shaded area with positive $\sigma(t)$ of the brown line in Fig.2(a). Namely, the atom-cavity coupling is the main physical reasons of the transition from Markovian to non-Markovian dynamics and enhancing the non-Markovianity in the dynamics process.

In Fig.3, we exhibit the curves of the non-Markovianity and the QSLT as functions of the coupling strength $\Omega$ when $s=1$ and $\frac{\omega_{c}}{\omega_{0}}=2$ for different coupling constant $\eta$, respectively. Fig.3(a) shows that $\mathcal{N}$ is always zero when $\Omega<\Omega_{c}$ and $\mathcal{N}$ will increase with $\Omega$ enlarging when $\Omega>\Omega_{c}$. Namely, there is a critical value $\Omega_{c}$ that $\mathcal{N}$ steeply increases from zero and the critical value is same for different coupling constant $\eta$. However, the increasing rate of $\mathcal{N}$ depends on the value of $\eta$, i.e., the smaller the coupling $\eta$, the stronger the non-Markovianity. The dependence of QSLT on the coupling $\Omega$ and $\eta$ is dotted in Fig.3(b), we find that $\tau_{Q S L T}$ is always equal $\tau$ when $\Omega<1.55 \omega_{0}$ and $\tau_{Q S L T}$ will decrease with $\Omega$ enlarging when $\Omega>1.55 \omega_{0}$. Namely, there is a critical value $\Omega_{c}$ of a sudden transition from no speedup to speedup and the critical value is same for different coupling constant $\eta$. But the decreasing rate of $\tau_{Q S L T}$ depends on the value of $\eta$, i.e., the smaller coupling $\eta$ is corresponding to the more obvious speedup process. This shows that, in addition to the atom-cavity coupling $\Omega$, the cavity-reservoir coupling $\eta$ can also regulate the non-Markovianity in the dynamics process and the speedup evolution process of the atom.

In Fig.4, we describe the dependence relation of the non-Markovianity and the QSLT on the coupling $\Omega$ and the cut-off frequency $\omega_{c}$ when $s=1$ and $\eta=0.9$. Fig.4(a) gives the non-Markovianity as a function of the coupling $\Omega$ for different values of $\omega_{c}$. If $\frac{\omega_{c}}{\omega_{0}}=2, \mathcal{N}$ is always zero when $\Omega<\Omega_{c}$ and $\mathcal{N}$ will increase with $\Omega$ enlarging when $\Omega>\Omega_{c}$. It should be noted that, if $\frac{\omega_{c}}{\omega_{0}}=1$ or $\frac{\omega_{c}}{\omega_{0}}=0.5$,
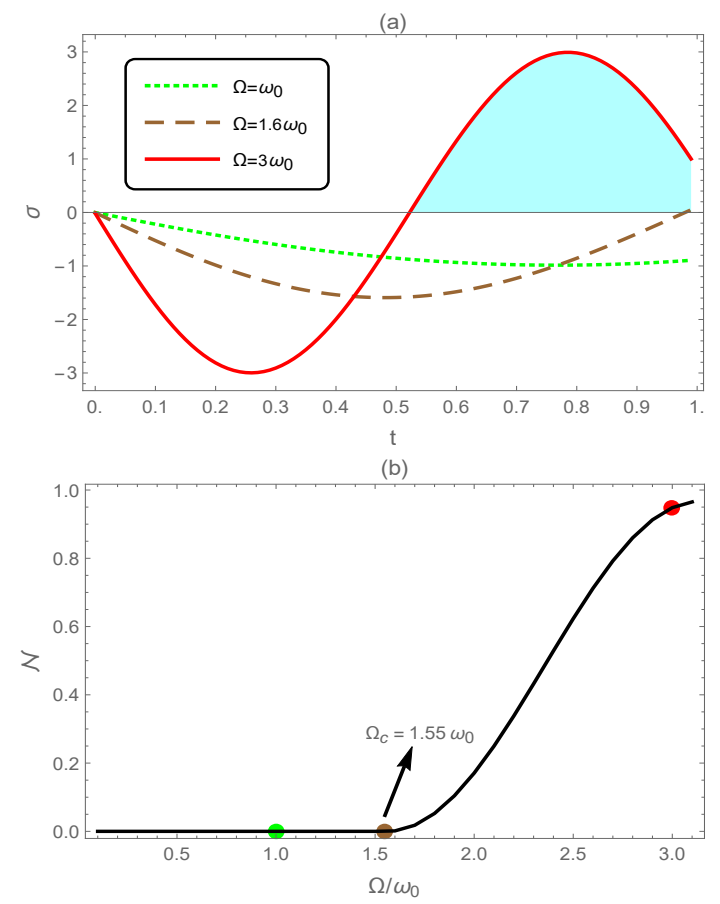

FIG. 2. (Color online)The dependence of the nonMarkovianity $\mathcal{N}$ on the derivative $\sigma(t)$ of the trace distance and the coupling strength $\Omega$ when $s=1$ (Ohmic spectrum) and $\frac{\omega_{c}}{\omega_{0}}=2$. (a)The curves of the derivative $\sigma$ for different $\Omega$ values: the green dotted line is an example with $\Omega=\omega_{0}$, the brown dashed line indicates an example with $\Omega=1.55 \omega_{0}$ and the red solid line represents $\Omega=3 \omega_{0}$ where the positive region of $\sigma(t)$ is shaded. (b)Non-Markovianity as a function of the coupling strength $\Omega$, in which the green dot is corresponding to the green line in (a), the brown dot corresponds to the brown line in (a) which is the transition point from Markovian to non-Markovian dynamics, and the red dot corresponds to the red line in (a). The other parameters are $\eta=0.1, \omega_{0}=1$ and $\omega_{c}=2$.

the non-Markovian dynamics occurring for $\Omega=0.1 \omega_{0}$ turns into Markovian and then back to non-Markovian by increasing $\Omega$, which such a behaviour has been also observed in different structured systems [72, 73]. But the critical value $\Omega_{c}$ is same for different values of $\omega_{c}$. Besides, we also find that, the smaller the value of $\frac{\omega_{c}}{\omega_{0}}$, the bigger the initial value of $\mathcal{N}$, and the bigger the value of $\mathcal{N}$ in areas with $\Omega>\Omega_{c}$. Fig.4(b) shows the QSLT as a function of the coupling $\Omega$ for different values of $\omega_{c}$. When $\frac{\omega_{c}}{\omega_{0}}=2, \tau_{Q S L T}$ is always equal $\tau$ when $\Omega<$ $\Omega_{c}$ and $\tau_{Q S L T}$ will decrease with $\Omega$ enlarging when $\Omega>$ $\Omega_{c}$. In particularly, when $\frac{\omega_{c}}{\omega_{0}}=1$ or $\frac{\omega_{c}}{\omega_{0}}=0.5, \tau_{Q S L T}$ will increase from a certain value to one and then again quickly decrease from one with $\Omega$ enlarging and there is a same critical value $\Omega_{c}$ for different values of $\omega_{c}$. In addition, we can see that, the smaller the value of $\frac{\omega_{c}}{\omega_{0}}$, the smaller the initial value of $\tau_{Q S L T}$, and the smaller the value of $\tau_{Q S L T}$ in areas with $\Omega>\Omega_{c}$. Therefore, not only the atom-cavity coupling $\Omega$ but also cut-off frequency $\omega_{c}$ can enhance the non-Markovianity in the dynamics 
(a)
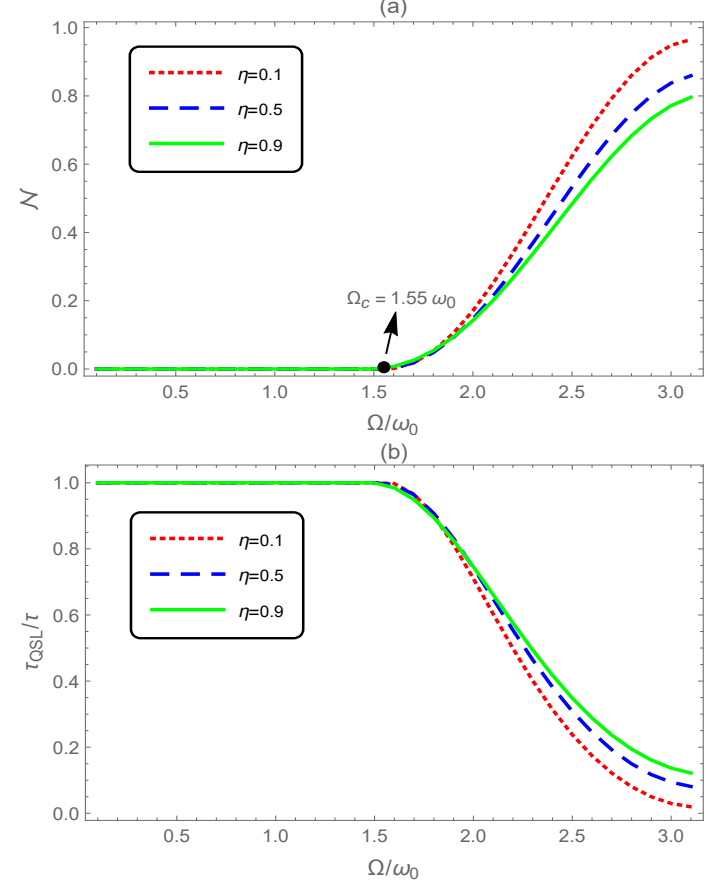

FIG. 3. (Color online)Non-Markovianity and QSLT as a function of the coupling strength $\Omega$ when $s=1$ and $\frac{\omega_{c}}{\omega_{0}}=2$ for different coupling constant $\eta$, respectively. $\eta=0 . \omega_{0}^{1}$, red dotted line; $\eta=0.5$, blue dashing line; $\eta=0.9$, green solid line. (a)Non-Markovianity as a function of $\Omega$; (b)QSLT as a function of $\Omega$. The other parameters are $\omega_{0}=1$ and $\omega_{c}=2$.

process and speed up the evolution of the atom.

The influences of $\Omega$ and $s$ on the non-Markovianity and the QSLT are shown in Fig.5. when $\eta=0.9$ and $\frac{\omega_{c}}{\omega_{0}}=2$. From Fig.5(a), we know that, for the Ohmic spectrum $(s=1)$ and the super-Ohmic spectrum $(s=3), \mathcal{N}$ is always zero when $\Omega<\Omega_{c}$ and $\mathcal{N}$ will increase with $\Omega$ enlarging when $\Omega>\Omega_{c}$, and their critical values are different. However, for the sub-Ohmic spectrum $\left(s=\frac{1}{2}\right)$, the non-Markovian dynamics occurring for $\Omega=0.1 \omega_{0}$ also turns into Markovian and then back to non-Markovian by increasing $\Omega$, and the critical value under the sub-Ohmic spectrum is obvious less than that under the Ohmic spectrum. From Fig.5(b), we discover that, for the Ohmic spectrum $(s=1)$ and the super-Ohmic spectrum $(s=3)$, $\tau_{Q S L T}$ is always zero when $\Omega<\Omega_{c}$ and $\tau_{Q S L T}$ will decrease with $\Omega$ enlarging when $\Omega>\Omega_{c}$, and their critical values are different. However, for the sub-Ohmic spectrum $\left(s=\frac{1}{2}\right), \tau_{Q S L T}$ will increase from 0.3 to one and then again quickly decrease from one with $\Omega$ enlarging, and the critical value under the sub-Ohmic spectrum is obvious less than that under the Ohmic spectrum. Namely, the atom-cavity coupling $\Omega$ and the Ohmicity parameter $s$ can effectively control the non-Markovianity in the dynamics process and speed up the evolution of the atom.

In the following, the physical interpretation of the results above is given. Because the cavity coupling with
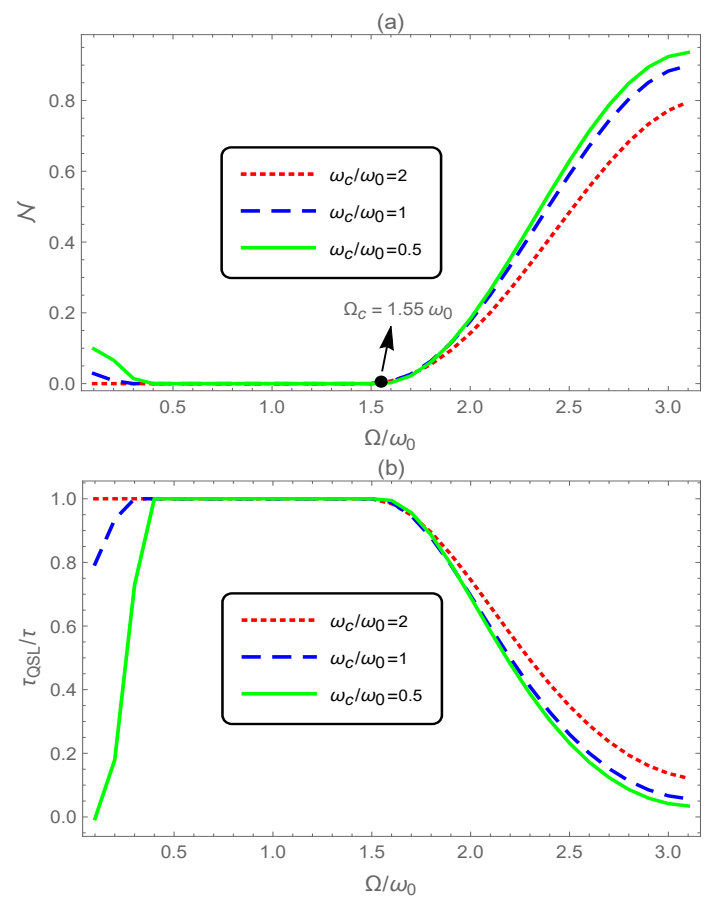

FIG. 4. (Color online)Non-Markovianity and QSLT as a function of the coupling strength $\Omega$ when $s=1$ (Ohmic spectrum) for different cutoff frequency $\omega_{c}$, respectively. $\frac{\omega_{c}}{\omega_{0}}=2$, red dotted line; $\frac{\omega_{c}}{\omega_{0}}=1$, blue dashing line; $\frac{\omega_{c}}{\omega_{0}}=0.5$, red solid line. (a)Non-Markovianity as a function of $\Omega$; (b)QSLT as a function of $\Omega$. The other parameters are $\eta=0.9$ and $\omega_{0}=1$.

the reservoir can be regarded as the environment of the atom, the energy and information can flow back from the environment to the atom through regulating the coupling strength $\Omega$. The larger the atom-cavity coupling $\Omega$, the more information the cavity flows back to the atom. Thus, the non-Markovianity will increase and the QSLT will decrease with $\Omega$ enlarging when $\Omega$ is bigger than the critical value. On the other hand, the influence of the cavity on the atom is obviously greater than that of the reservoir on the atom, so the critical value of sudden transition is mainly determined by $\Omega$. From Eq. (11), we know that a smaller value of $\eta$ corresponds to a longer correlation time of the reservoir thus the nonMarkovianity $\mathcal{N}$ is bigger and the QSLT is smaller. Moreover, the smaller value of $\frac{\omega_{c}}{\omega_{0}}$ corresponds to the less overlap of the spectrum of the reservoir with the frequency of the cavity, that is, the reservoir is more effectively adiabatic and the non-Markovian effect is more obvious and the evolution of the atom is quicker. The smaller the Ohmicity parameter $s$ is, the smaller the peak and the width of the Ohmic spectral density are, the more obvious the non-Markovian effect is. So the smaller value of $s$ will lead to the larger non-Markovianity and the smaller QSLT. Besides, Eq. 24) shows that the information flows irreversibly from the atom to the environment so that the atom evolves at the actual speed and the QSLT is equal to the actual evolution time when $\mathcal{N}=0$. The informa- 

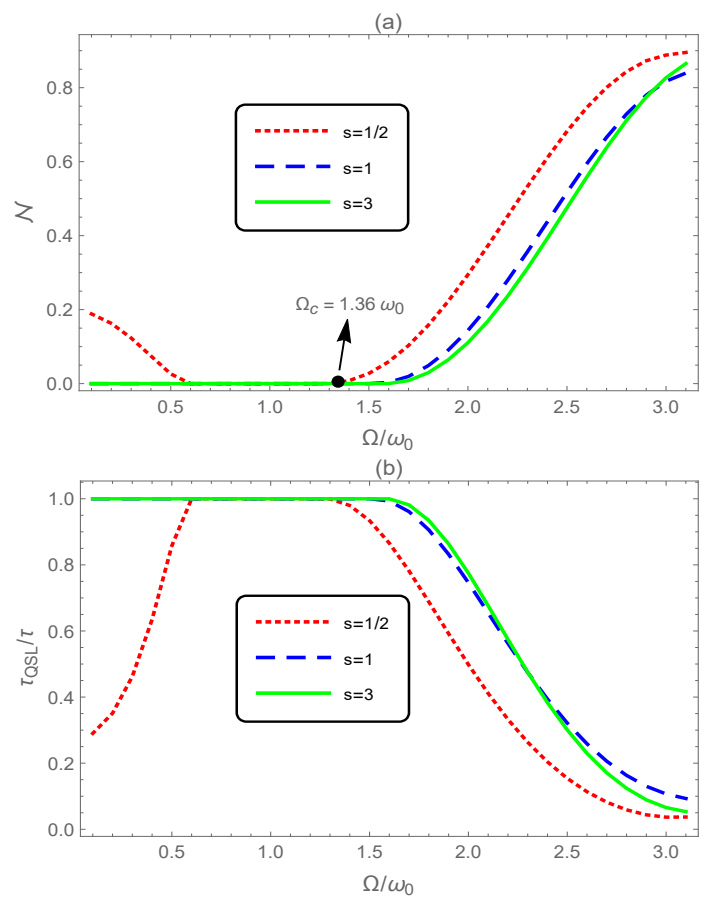

FIG. 5. (Color online)Non-Markovianity and QSLT as a function of the coupling strength $\Omega$ for different Ohmicity parameter $s$, respectively. $s=\frac{1}{2}$ (sub-Ohmic spectrum), red dotted line; $s=1$ (Ohmic spectrum), blue dashing line; $s=3$ (superOhmic spectrum), green solid line. (a)Non-Markovianity as a function of $\Omega$; (b)QSLT as a function of $\Omega$. The other parameters are $\eta=0.6, \omega_{0}=1$ and $\omega_{c}=2$.

tion flows back from the environment to the atom thus the atom evolution is accelerated and the QSLT is smaller than the actual evolution time when $\mathcal{N}>0$.

\section{CONCLUSION}

In summary, we have investigated the nonMarkovianity and the QSLT of the atom in JaynesCummings model coupling with the Ohmic reservoir when the total excitation number is $n=1$. We have obtained the non-Markovianity characterized by using the probability of the atomic excited state and the negative decoherence rate in the time-local master equation (see Eq. (21)), which also showed that the non-Markovianity can be explained reasonably by the negative decoherence rate, namely, the dynamical process is non-Markovian if the decoherence rate is negative 74. We have also studied in detail the influence of the atom-cavity coupling and the reservoir parameters on the non-Markovianity and the QSLT. The results have showed that, the atom-cavity coupling is the main physical reasons of the transition from Markovian to non-Markovian dynamics and the transition from no speedup to speedup process, and the critical value of this sudden transition only depends on the Ohmicity parameter. The appropriate reservoir parameters, such as the cavity-reservoir coupling $\eta$, the cut-off frequency $\omega_{c}$ and the Ohmicity parameter $s$, can improve the non-Markovianity in the dynamics process and speed up the evolution of the atom. In addition, we have also found that the non-Markovian dynamics occurring for $\Omega=0.1 \omega_{0}$ turns into Markovian and then back to non-Markovian by increasing $\Omega$ when $\frac{\omega_{c}}{\omega_{0}}=1, \frac{\omega_{c}}{\omega_{0}}=0.5$ or $s=\frac{1}{2}$ ( the sub-Ohmic spectrum).

In this work, only zero temperature reservoir is considered. If the reservoir is at nonzero temperature, from Eq. (4), we can see that, the quantum coherence of the atom-cavity and the populations of the states $\left|\varphi_{1, \mp}\right\rangle$ will increase a little under the effect of thermal reservoir. The non-Markovianity and the QSLT of the atom will be different from zero temperature case. The detailed influence of nonzero temperature on quantum effect will be presented in our next work. These results will provide interesting perspectives for future applications of open quantum systems in quantum physics 75,78 .

\section{ACKNOWLEDGMENTS}

This work was supported by the National Natural Science Foundation of China (Grant No 11374096) and the Doctoral Science Foundation of Hunan Normal University, China.
[1] Edward Brian Davies. Quantum theory of open systems. Academic Press, 1976.

[2] Goran Lindblad. On the generators of quantum dynamical semigroups. Communications in Mathematical Physics, 48(2):119-130, 1976.

[3] Andrzej Kossakowski. On quantum statistical mechanics of non-hamiltonian systems. Reports on Mathematical Physics, 3(4):247-274, 1972.

[4] Vittorio Gorini, Andrzej Kossakowski, and Ennackal Chandy George Sudarshan. Completely positive dynamical semigroups of n-level systems. Journal of Mathematical Physics, 17(5):821-825, 1976.
[5] Frank Verstraete, Michael M. Wolf, and J. Ignacio Cirac. Quantum computation and quantum-state engineering driven by dissipation. Nature physics, 5(9):633-636, 2009.

[6] Ángel Rivas, Susana F. Huelga, and Martin B. Plenio. Quantum non-markovianity: characterization, quantification and detection. Reports on Progress in Physics, 77(9):094001, 2014.

[7] Michael Marc Wolf, J. Eisert, T. S. Cubitt, and J. Ignacio Cirac. Assessing non-markovian quantum dynamics. Physical review letters, 101(15):150402, 2008.

[8] Heinz-Peter Breuer, Elsi-Mari Laine, and Jyrki Piilo. 
Measure for the degree of non-markovian behavior of quantum processes in open systems. Physical review letters, 103(21):210401, 2009.

[9] Ángel Rivas, Susana F. Huelga, and Martin B. Plenio. Entanglement and non-markovianity of quantum evolutions. Physical review letters, 105(5):050403, 2010.

[10] Shunlong Luo, Shuangshuang Fu, and Hongting Song. Quantifying non-markovianity via correlations. Physical Review A, 86(4):044101, 2012.

[11] Hao-Sheng Zeng, Ning Tang, Yan-Ping Zheng, and Guo-You Wang. Equivalence of the measures of nonmarkovianity for open two-level systems. Physical Review A, 84(3):032118, 2011.

[12] Zhi He, Hao-Sheng Zeng, Yan Li, Qiong Wang, and Chunmei Yao. Non-markovianity measure based on the relative entropy of coherence in an extended space. Physical Review A, 96(2):022106, 2017.

[13] Carlos Pineda, Thomas Gorin, David Davalos, Diego A. Wisniacki, and Ignacio García-Mata. Measuring and using non-markovianity. Physical Review A, 93(2):022117, 2016.

[14] Pablo Matías Poggi, Fernando Cesar Lombardo, and Diego Ariel Wisniacki. Driving-induced amplification of non-markovianity in open quantum systems evolution. EPL (Europhysics Letters), 118(2):20005, 2017.

[15] Zhi He, Chunmei Yao, Qiong Wang, and Jian Zou. Measuring non-Markovianity based on local quantum uncertainty. Phys. Rev. A, 90(4):042101, 2014.

[16] Ruari McCloskey and Mauro Paternostro. NonMarkovianity and system-environment correlations in a microscopic collision model. Phys. Rev. A, 89(5):052120, 2014.

[17] Salvatore Lorenzo, Francesco Plastina, and Mauro Paternostro. Geometrical characterization of nonMarkovianity. Phys. Rev. A, 88(2):020102, 2013.

[18] Ali Mortezapour, Mahdi Ahmadi Borji, and Rosario Lo Franco. Protecting entanglement by adjusting the velocities of moving qubits inside non-Markovian environments. Laser Phys. Lett., 14(5):055201, 2017.

[19] Yu Liu, Hong-Mei Zou, and Mao-Fa Fang. Quantum coherence and non-Markovianity of an atom in a dissipative cavity under weak measurement. Chinese Phys. B, 27(1):010304, 2018.

[20] Hossein Gholipour, Ali Mortezapour, Farzam Nosrati, and Rosario Lo Franco. Quantumness and memory of one qubit in a dissipative cavity under classical control. Ann. Phys., 414:168073, 2020.

[21] Hossein Rangani Jahromi, Kobra Mahdavipour, Mahshid Khazaei Shadfar, and Rosario Lo Franco. Measuring nonMarkovian effects of quantum processes through HilbertSchmidt speed. arXiv, 2003.12681, 2020.

[22] H Rangani Jahromi, Morteza Amini, and M Ghanaatian. Multiparameter estimation, lower bound on quantum Fisher information, and non-Markovianity witnesses of noisy two-qubit systems. Quantum Inf. Process., 18(11):338, 2019.

[23] Philipp Strasberg and Massimiliano Esposito. NonMarkovianity and negative entropy production rates. Phys. Rev. E, 99(1):012120, 2019.

[24] Zhi He, Hao-Sheng Zeng, Yan Li, Qiong Wang, and Chunmei Yao. Non-Markovianity measure based on the relative entropy of coherence in an extended space. Phys. Rev. A, 96(2):022106, 2017.
[25] Himadri Shekhar Dhar, Manabendra Nath Bera, and Gerardo Adesso. Characterizing non-Markovianity via quantum interferometric power. Phys. Rev. A, 91(3):032115, 2015.

[26] Inés de Vega and Daniel Alonso. Dynamics of nonmarkovian open quantum systems. Reviews of Modern Physics, 89(1):015001, 2017.

[27] Heinz-Peter Breuer, Elsi-Mari Laine, Jyrki Piilo, and Bassano Vacchini. Colloquium: Non-markovian dynamics in open quantum systems. Reviews of Modern Physics, 88(2):021002, 2016.

[28] David Davalos and Carlos Pineda. Quantum nonmarkovianity and localization. Physical Review A, 96(6):062127, 2017.

[29] Shang Yu, Yi-Tao Wang, Zhi-Jin Ke, Wei Liu, Yu Meng, Zhi-Peng Li, Wen-Hao Zhang, Geng Chen, Jian-Shun Tang, and Chuan-Feng Li. Experimental investigation of spectra of dynamical maps and their relation to nonmarkovianity. Physical review letters, 120(6):060406, 2018.

[30] J. Anandan and Yakir Aharonov. Geometry of quantum evolution. Physical review letters, 65(14):1697, 1990.

[31] Lev Vaidman. Minimum time for the evolution to an orthogonal quantum state. American journal of physics, 60(2):182-183, 1992.

[32] Shunlong Luo. How fast can a quantum state evolve into a target state? Physica D: Nonlinear Phenomena, 189(12):1-7, 2004.

[33] Seth Lloyd. Ultimate physical limits to computation. Nature, 406(6799):1047-1054, 2000.

[34] Vittorio Giovannetti, Seth Lloyd, and Lorenzo Maccone. Quantum metrology. Physical review letters, 96(1):010401, 2006.

[35] Philip J. Jones and Pieter Kok. Geometric derivation of the quantum speed limit. Physical Review A, 82(2):022107, 2010.

[36] Marcin Zwierz. Comment on "geometric derivation of the quantum speed limit". Physical Review A, 86(1):016101, 2012.

[37] Zhen-Yu Xu, Shunlong Luo, W. L. Yang, Chen Liu, and Shiqun Zhu. Quantum speedup in a memory environment. Physical Review A, 89(1):012307, 2014.

[38] Xiangji Cai and Yujun Zheng. Quantum dynamical speedup in a nonequilibrium environment. Physical Review $A, 95(5): 052104,2017$.

[39] Francesco Campaioli, Felix A. Pollock, Felix C. Binder, and Kavan Modi. Tightening quantum speed limits for almost all states. Physical review letters, 120(6):060409, 2018.

[40] L. Mandelstam and I. E. Tamm. The energy-time uncertainty relation in non-relativistic quantum mechanics. Izv. Akad. Nauk SSSR, 9:122, 1945.

[41] Norman Margolus and Lev B. Levitin. The maximum speed of dynamical evolution. Physica D: Nonlinear Phenomena, 120(1-2):188-195, 1998.

[42] Sebastian Deffner and Eric Lutz. Quantum speed limit for non-markovian dynamics. Physical review letters, 111(1):010402, 2013.

[43] Márcio M. Taddei, Bruno M. Escher, Luiz Davidovich, and de Matos Filho, Ruynet L. Quantum speed limit for physical processes. Physical review letters, 110(5):050402, 2013.

[44] A. Del Campo, I. L. Egusquiza, Martin B. Plenio, and Susana F. Huelga. Quantum speed limits in open system 
dynamics. Physical review letters, 110(5):050403, 2013.

[45] Shao-xiong Wu and Chang-shui Yu. Quantum speed limit for a mixed initial state. Physical Review A, 98(4):042132, 2018.

[46] Ying-Jie Zhang, Wei Han, Yun-Jie Xia, Jun-Peng Cao, and Heng Fan. Quantum speed limit for arbitrary initial states. Scientific reports, 4(1):1-6, 2014.

[47] Shao-xiong Wu, Yang Zhang, Chang-shui Yu, and Heshan Song. The initial-state dependence of the quantum speed limit. Journal of Physics A: Mathematical and Theoretical, 48(4):045301, 2014.

[48] Junjie Liu, Dvira Segal, and Gabriel Hanna. Hybrid quantum-classical simulation of quantum speed limits in open quantum systems. Journal of Physics A: Mathematical and Theoretical, 52(21):215301, 2019.

[49] Marco Cianciaruso, Sabrina Maniscalco, and Gerardo Adesso. Role of non-markovianity and backflow of information in the speed of quantum evolution. Physical Review A, 96(1):012105, 2017.

[50] Bahram Ahansaz and Abbas Ektesabi. Quantum speedup, non-Markovianity and formation of bound state. Sci. Rep., 9(1):14946, 2019.

[51] Zhen-Yu Xu, Shunlong Luo, W L Yang, Chen Liu, and Shiqun Zhu. Quantum speedup in a memory environment. Phys. Rev. A, 89(1):012307, 2014.

[52] Hai-Bin Liu, W. L. Yang, Jun-Hong An, and Zhen-Yu $\mathrm{Xu}$. Mechanism for quantum speedup in open quantum systems. Physical Review A, 93(2):020105, 2016.

[53] Nicolás Mirkin, Fabricio Toscano, and Diego A Wisniacki. Quantum-speed-limit bounds in an open quantum evolution. Phys. Rev. A, 94(5):052125, 2016.

[54] Jing Wang, Y. N. Wu, and Z. Y. Xie. Role of flow of information in the speedup of quantum evolution. Scientific reports, 8(1):1-10, 2018.

[55] Sebastian Deffner and Eric Lutz. Quantum Speed Limit for Non-Markovian Dynamics. Phys. Rev. Lett., 111(1):010402, 2013.

[56] Kai Xu, Ying-Jie Zhang, Yun-Jie Xia, Z D Wang, and Heng Fan. Hierarchical-environment-assisted nonMarkovian speedup dynamics control. Phys. Rev. A, 98(2):022114, 2018.

[57] Ying-Jie Zhang, Wei Han, Yun-Jie Xia, Jun-Peng Cao, and Heng Fan. Classical-driving-assisted quantum speedup. Physical Review A, 91(3):032112, 2015.

[58] Hong-Mei Zou, Jianhe Yang,Danping Lin ,Mao-Fa Fang. Quantum speed-up process of atom in dissipative cavity. Journal of Physics B: Atomic, Molecular and Optical Physics, 2020.

[59] Edwin T. Jaynes and Frederick W. Cummings. Comparison of quantum and semiclassical radiation theories with application to the beam maser. Proceedings of the IEEE, 51(1):89-109, 1963.

[60] Bruce W. Shore and Peter L. Knight. The jaynescummings model. Journal of Modern Optics, 40(7):11951238, 1993.

[61] M. Scala, B. Militello, A. Messina, Jyrki Piilo, and Sabrina Maniscalco. Microscopic derivation of the jaynescummings model with cavity losses. Physical Review A, 75(1):013811, 2007.

[62] Hong-Mei Zou and Mao-Fa Fang. Analytical solution and entanglement swapping of a double jaynes-cummings model in non-markovian environments. Quantum Information Processing, 14(7):2673-2686, 2015.

[63] Herbert Spohn and Joel L Lebowitz. Irreversible thermodynamics for quantum systems weakly coupled to thermal reservoirs. Adv. Chem. Phys, 38:109-142, 1978.

[64] Ming-Liang $\mathrm{Hu}$ and Heng Fan. Quantum-memoryassisted entropic uncertainty principle, teleportation, and entanglement witness in structured reservoirs. Physical Review A, 86(3):032338, 2012.

[65] Anthony J. Leggett, SDAFMGA Chakravarty, Alan T. Dorsey, Matthew P. A. Fisher, Anupam Garg, and Wilhelm Zwerger. Dynamics of the dissipative two-state system. Reviews of Modern Physics, 59(1):1, 1987.

[66] Claudia Benedetti, Fahimeh Salari Sehdaran, Mohammad H. Zandi, and Matteo G. A. Paris. Quantum probes for the cutoff frequency of ohmic environments. Physical Review A, 97(1):012126, 2018.

[67] Hong-Mei Zou, Mao-Fa Fang, You-Neng Guo, and BaiYuan Yang. Quantum discord of the two-atom system in non-markovian environments. Physica Scripta, 90(3):035104, 2015.

[68] Jens Eckel, John Henry Reina, and Michael Thorwart. Coherent control of an effective two-level system in a nonmarkovian biomolecular environment. New Journal of Physics, 11(8):085001, 2009.

[69] Wei Cui, Zairong Xi, and Yu Pan. Non-markovian entanglement dynamics between two coupled qubits in the same environment. Journal of Physics A: Mathematical and Theoretical, 42(15):155303, 2009.

[70] Heinz-Peter Breuer and Francesco Petruccione. The theory of open quantum systems. Oxford University Press on Demand, 2002.

[71] Tillmann Baumgratz, Marcus Cramer, and Martin B. Plenio. Quantifying coherence. Physical review letters, 113(14):140401, 2014.

[72] Zhong-Xiao Man, Yun-Jie Xia, and Rosario Lo Franco. Harnessing non-markovian quantum memory by environmental coupling. Physical Review A, 92(1):012315, 2015.

[73] Zhong-Xiao Man, Yun-Jie Xia, and Rosario Lo Franco. Cavity-based architecture to preserve quantum coherence and entanglement. Scientific reports, 5:13843, 2015.

[74] Michael J W Hall, James D Cresser, Li Li, and Erika Andersson. Canonical form of master equations and characterization of non-Markovianity. Phys. Rev. A, 89(4):042120, 2014.

[75] Benjamin T. H. Varcoe, Simon Brattke, Matthias Weidinger, and Herbert Walther. Preparing pure photon number states of the radiation field. Nature, 403(6771):743-746, 2000.

[76] Daniel Jonathan and Martin B. Plenio. Light-shiftinduced quantum gates for ions in thermal motion. Physical review letters, 87(12):127901, 2001.

[77] J. Q. You and Franco Nori. Atomic physics and quantum optics using superconducting circuits. Nature, 474(7353):589-597, 2011.

[78] Steven Prawer and Andrew D. Greentree. Diamond for quantum computing. Science, 320(5883):1601-1602, 2008. 